\title{
Avaliação do Seguimento de Mulheres com Exames Citopatológicos Alterados de acordo com as Condutas Preconizadas pelo Ministério da Saúde do Brasil em Goiânia, Goiás
} Follow-up Evaluation of Women with Abnormal Cytopathological Tests According to the Procedures Recommended by the Ministry of Health of Brazil in Goiania city, State of Goias

Evaluación de Seguimiento de Mujeres con Exámenes Citopatológicos Alterados de acuerdo a las Conductas Recomendadas por el Ministerio de Salud de Brasil en la Ciudad de Goiania, Estado de Goias

\author{
Eduardo Silva Araújo'; Flávia Moreira Barbosa² ; Cinara Zago Silveira Ázara3 ; Tatyana Xavier Almeida Matteucci Ferreira ${ }^{4}$ S Suelene Brito do \\ Nascimento Tavares ${ }^{5}$; Rita Goreti Amaral ${ }^{6}$
}

\begin{abstract}
Resumo
Introduçáo: $\mathrm{O}$ adequado seguimento para mulheres com exames citopatológicos alterados é importante para o tratamento das lesōes precursoras em sua fase inicial. Objetivo: Avaliar se as mulheres atendidas nas Unidades de Atenção Básica com exames citopatológicos alterados foram submetidas adequadamente às condutas preconizadas pelo Ministério da Saúde. Método: O estudo analisou 12.208 resultados de exames citopatológicos de mulheres atendidas nas Unidades de Atenção Básica à Saúde em Goiânia, no período de 2006 a 2008, através dos prontuários das Unidades de Atenção Básica e Média Complexidade. O programa Epi-info 3.5.1 foi utilizado para análise dos dados. Resultados: Dos 12.208 resultados analisados, 90,74\% foram classificados como negativos, 1,88\% como insatisfatórios, 4,74\% como lesões menos graves, e 2,43\% como lesóes de média gravidade. Do total de 579 mulheres com exames citopatológicos classificados como células escamosas atípicas de significado indeterminado, possivelmente não neoplásicas/lesão intraepitelial de baixo grau, 41,62\% realizaram citopatologia de seguimento, 14,85\% e 10,19\% realizaram colposcopia e biópsia, respectivamente. Das 297 mulheres com resultados citopatológicos classificados como células escamosas atípicas de significado indeterminado, não se pode excluir lesão de alto grau/lesão intraepitelial de alto grau, 42,76\% realizaram citopatologia de seguimento, 35,02\% e 28,96\% realizaram colposcopia e biópsia, respectivamente. Das 24 mulheres com resultado citopatológico classificado como células glandulares atípicas/adenocarcinoma invasor, 20,83\% realizaram citopatologia de seguimento, e 16,67\% e 12,50\% das mulheres realizaram colposcopia e biópsia, respectivamente. Conclusáo: A maioria das mulheres com exames citopatológicos alterados possivelmente não foi submetida às condutas preconizadas conforme as recomendaçôes do Ministério da Saúde.

Palavras-chave: Feminino; Neoplasias do Colo do Útero-diagnóstico; Neoplasias do Colo do Útero-epidemiologia; Atenção Primária à Saúde; Programas de Rastreamento-utilização; Brasil-epidemiologia
\end{abstract}

\footnotetext{
${ }^{1}$ Acadêmico do Curso de Farmácia da Universidade Federal de Goiás (UFG). Goiânia (GO), Brasil. E-mail: eduardos.araujo@hotmail.com.

${ }^{2}$ Acadêmica do Curso de Farmácia da UFG. Goiânia (GO), Brasil. E-mail: flavia.moreirabarbosa@gmail.com.

${ }^{3}$ Biomédica Citologista do Laboratório de Monitoramento Externo da Qualidade da Faculdade de Farmácia da UFG. Mestre em Ciências da Saúde pela UFG. Goiânia (GO), Brasil. E-mail: cinarazago@hotmail.com.

${ }^{4}$ Farmacêutica do Hospital das Clínicas da UFG. Doutora em Ciências da Saúde pela UFG. Goiânia (GO), Brasil. E-mail: tatymatteucci@gmail.com.

${ }^{5}$ Biomédica Citologista do Laboratório de Monitoramento Externo da Qualidade da Faculdade de Farmácia da UFG. Doutora em Ciências da Saúde pela UFG. Goiânia (GO), Brasil. E-mail: suelenetavares@gmail.com.

${ }^{6}$ Farmacêutica. Doutora em Tocoginecologia pela Universidade Estadual de Campinas. Professora da Faculdade de Farmácia da UFG. Goiânia (GO), Brasil. E-mail: ritagoreti26@gmail.com.

Endereço para correspondência: Rita Goreti Amaral. Avenida Belo Horizonte, Quadra 39, Lote 4, Setor Jaó. Goiânia (GO), Brasil. CEP: 74.673-020. E-mail: ritagoreti26@gmail.com.
} 


\section{INTRODUÇÃO}

O câncer do colo do útero no Brasil é um dos principais problemas de saúde pública enfrentado pelas mulheres, representando a segunda causa de morte nessa população, ficando atrás apenas do câncer de mama e de pele do tipo não melanoma ${ }^{1}$. O número de casos novos desse tipo de câncer para o Brasil no ano de 2013 foi estimado em 17.540, com um risco de 17 casos a cada 100 mil mulheres ${ }^{2}$.

A ocorrência do câncer do colo do útero está intimamente relacionada à infecção pelo Papiloma Vírus Humano (HPV), transmitido sexualmente ${ }^{3}$. Além disso, outros fatores também podem contribuir para o desenvolvimento desse câncer, como o tabagismo, o uso de contraceptivos orais, outras doenças sexualmente transmissíveis e deficiências nutricionais ${ }^{4}$.

O exame citopatológico é eficiente e muito utilizado para detectar as lesôes precursoras do câncer do colo do útero, principalmente em fases iniciais, quando o tratamento apresenta alto índice de cura, diminuindo a mortalidade, tornando baixo o custo efetivo ${ }^{5}$. Estima-se uma redução de cerca de $80 \%$ da mortalidade por esse câncer por meio do rastreamento de mulheres na faixa etária de 25 a 64 anos com o diagnóstico precoce e o tratamento das lesôes precursoras ${ }^{6}$. Esse rastreamento é fundamental para que se evite o aparecimento de novos casos de câncer, os quais exigem tratamentos mais complexos e dispendiosos ${ }^{1}$. Para tanto, é necessário garantir a organização, a integralidade e a qualidade do programa de rastreamento ${ }^{7}$.

No Sistema Único de Saúde (SUS), as açôes de prevenção e controle desse câncer são estruturadas a partir das Unidades de Atençáo Básica de Saúde (UABS) com colaboraçáo dos laboratórios de citopatologia, histopatologia e hospitais especializados ${ }^{7}$. Essas ações são monitoradas pelo Sistema de Informaçóes do Câncer do Colo do Útero (SISCOLO), que compreende um sistema destinado ao armazenamento de dados sobre identificação da mulher, informaçôes demográficas, epidemiológicas e dos exames citopatológicos e histopatológicos realizados no SUS 8 .

Portanto, para que tenha sucesso, é necessário que o programa de rastreamento seja organizado e definido como um conjunto de açóes programadas, com população e periodicidade específicas ${ }^{9,10}$. Entretanto, no Brasil, ocorre a procura ocasional dos serviços de saúde, determinada por razóes diversas que não o rastreamento do câncer do colo do útero, o que leva à realização de controles não relacionados com as normas estabelecidas. Essa procura ocasional é designada rastreamento oportunístico, o que não é eficiente em reduzir as taxas de incidência e mortalidade do câncer do colo do útero ${ }^{9,10,11}$. O rastreamento oportunístico tem como característica a baixa cobertura e o super-rastreamento de um pequeno grupo de mulheres, tornando-se menos custo-efetivo ${ }^{10,12}$.

Pode ocorrer também uma orientação errônea por parte dos profissionais de saúde. Essa diferença entre a prática vigente e as normas do Ministério da Saúde (MS) é uma das dificuldades para o sucesso das ações do rastreamento ${ }^{13}$.

Diante disso, o objetivo desse estudo foi avaliar se as mulheres atendidas nas UABS com exames citopatológicos alterados foram submetidas adequadamente às condutas preconizadas pelo MS.

\section{MÉTODO}

Estudo descritivo realizado na Faculdade de Farmácia da Universidade Federal de Goiás (UFG), Goiânia, Goiás, Brasil, como parte do projeto de pesquisa intitulado "Controle interno da qualidade dos exames citopatológicos do colo do útero: pré-escrutínio rápido versus revisão rápida de $100 \% "$, aprovado pelo Comitê de Ética dessa instituiçáo sob o protocolo no 001/09 (COEP-UFG).

Este estudo teve como base os resultados dos exames citopatológicos alterados do colo do útero da população feminina usuária do SUS, atendida nas UABS do município de Goiânia, realizados no Laboratório Rômulo Rocha, no período de março de 2006 a maio de 2008, totalizando 12.208 exames analisados.

Os exames citopatológicos alterados foram identificados por meio do SISCOLO. Foram incluídos somente os resultados de mulheres que já iniciaram a atividade sexual ${ }^{14}$; maiores de 18 anos na data da coleta.

Para obtenção das informaçôes de seguimento das mulheres, fez-se um levantamento por meio dos prontuários nas UABS e nas Unidades de Média Complexidade (UMC), para as quais as mulheres foram encaminhadas, quando tinham o resultado do exame citopatológico alterado. Posteriormente, avaliou-se a conformidade do seguimento tendo como referência a conduta clínica preconizada pelo Instituto Nacional de Câncer José Alencar Gomes da Silva (INCA)/MS ${ }^{15}$.

Os resultados dos exames citopatológicos foram categorizados de acordo com a Nomenclatura Brasileira para Laudos Cervicais ${ }^{15} \mathrm{em}$ : negativo para neoplasia, células escamosas atípicas de significado indeterminado, possivelmente não neoplásicas (ASC-US); células escamosas atípicas de significado indeterminado, não se pode excluir lesão de alto grau (ASC-H); lesão intraepitelial de baixo grau (LSIL); lesão intraepitelial de alto grau (HSIL); células glandulares atípicas (AGC); adenocarcinoma in situ (Adeno in situ); e carcinomas invasores, principalmente, carcinoma escamoso invasor (Ca inv) e adenocarcinoma invasor (Adeno inv), entre outros. 
O MS recomenda que as mulheres com exame citopatológico classificado como ASC-US ou LSIL devem repetir o exame citopatológico após seis meses nas UABS. As mulheres com exame citopatológico classificado como ASC-H, HSIL, carcinoma invasor, CGA, adeno in situ ou adenocarcinoma invasor devem ser encaminhadas à UMC para se submeterem à colposcopia e à confirmaçáo histológica e, se necessário, deverão ser encaminhadas à Unidade de Alta Complexidade (UAC) para receberem tratamento de acordo com as condutas específicas recomendadas ${ }^{15}$.

O resultado da colposcopia foi classificado de acordo com a classificação colposcópica da Federação Internacional de Patologia Cervical, de 2003, em: insatisfatória, satisfatória, normal e anormal ${ }^{16}$.

O resultado da biópsia foi classificado em: normal, cervicite crônica e metaplasia escamosa, neoplasia intraepitelial grau I (NIC I), neoplasia intraepitelial grau II/III (NIC II/III), carcinoma escamoso invasivo, adenocarcinoma in situ e adenocarcinoma invasor ${ }^{17}$.

Considerou-se seguimento ignorado os casos que não tinham informaçôes no prontuário.

Para a análise dos dados, os resultados dos exames citopatológicos foram agrupados e categorizados de acordo com a conduta clínica preconizada e a gravidade da lesáo da seguinte maneira: os resultados classificados como ASC-US ou LSIL foram categorizados como ASC-US/ LSIL (lesões menos graves); os classificados como ASC-H ou HSIL foram categorizados como ASC-H/HSIL (lesóes de média gravidade); os classificados como AGC e adenocarcinoma in situ foram categorizados como AGC/ adeno in situ (lesões mais graves), e carcinoma escamoso invasor e adenocarcinoma invasor foram categorizados como Ca inv/Adeno inv (lesôes gravíssimas) ${ }^{15}$.

O programa Epi-info 3.5.1 foi utilizado para o processamento e a análise dos dados. A distribuição das variáveis: resultados dos exames citopatológicos, resultados dos exames colposcópicos e da biópsia, foram calculadas utilizando-se as frequências absolutas e relativas ${ }^{18}$.

\section{RESULTADOS}

O estudo mostrou que, dos 12.208 resultados analisados, $11.078(90,74 \%)$ foram classificados como negativo, 230 (1,88\%) como insatisfatório, 579 (4,74\%) como lesôes menos graves ASC-US/LSIL. As lesōes de média gravidade ASC-H/HSIL totalizaram 297 (2,43\%) dos esfregaços analisados. Já as lesōes mais graves AGC/ adeno in situ totalizaram 24 (0,20\%) e as lesôes gravíssimas Ca inv/ adeno inv não obtiveram resultados (Tabela1).

Do total de 579 mulheres com exames citopatológicos alterados classificados como ASC-US/LSIL, 241 (41,62\%) repetiram a citopatologia em seis meses, sendo que 338 $(58,38 \%)$ tiveram seguimento ignorado (Tabela 2$)$.
Verificou-se ainda que das 297 mulheres com resultados citopatológicos classificados como ASC-H/HSIL, 104 $(35,02 \%)$ e $86(28,96 \%)$ realizaram a colposcopia e a biópsia, respectivamente, sendo que 193(64,98\%) tiveram seguimento ignorado e $127(42,76 \%)$ repetiram a citopatologia em seis meses (Tabela 3 ).

Das 24 mulheres com resultado do exame citopatológico classificado como AGC/adeno in situ, apenas quatro $(16,67 \%)$ e três $(12,50 \%)$ mulheres realizaram a colposcopia e a biópsia, respectivamente, sendo que 20 $(83,33)$ tiveram seguimento ignorado, e cinco $(20,83 \%)$ repetiram a citopatologia em seis meses (Tabela 3 ).

Tabela 1. Resultados dos exames citopatologicos de 12.208 mulheres atendidas nas Unidades Atenção Básicas de Saúde, no município de Goiânia, no período de março de 2006 a maio de 2008

\begin{tabular}{l|c|c} 
Diagnóstico & $\begin{array}{c}\text { Frequência } \\
(\mathrm{n})\end{array}$ & $\begin{array}{c}\text { Porcentagem } \\
(\%)\end{array}$ \\
\hline Insatisfatório & 230 & 1,88 \\
\hline Negativo & 11.078 & 90,74 \\
\hline ASC-US & 277 & 2,27 \\
\hline ASC-H & 121 & 0,99 \\
\hline LSIL & 302 & 2,47 \\
\hline HSIL & 176 & 1,44 \\
\hline AGC & 24 & 0,20 \\
\hline Adeno in situ & 0 & 0,00 \\
\hline Ca inv & 0 & 0,00 \\
\hline Adeno inv & 0 & 0,00 \\
\hline Total & 12.208 & 100 \\
\hline
\end{tabular}

ASC-US: células escamosas atípicas de significado indeterminado, possivelmente não neoplásicas; ASC-H: células escamosas atípicas de significado indeterminado, não podendo excluir lesão de alto grau; LSIL: lesão intraepitelial de baixo grau; HSIL: lesāo intraepitelial de alto grau; AGC: células glandulares atípicas; adeno in situ: adenocarcinoma in situ; Ca inv: carcinoma escamoso invasor; adeno inv: adenocarcinoma invasor.

\section{DISCUSSÃo}

Os resultados deste estudo mostraram que, das mulheres com exames citopatológicos classificados como ASC-US/LSIL, 241 (41,62\%) repetiram o exame citopatológico em seis meses conforme as recomendaçóes do $\mathrm{MS}^{15}$ e aproximadamente $15,00 \%$ foram submetidas à colposcopia e biópsia. Situação semelhante foi observada em estudo de Bueno ${ }^{19}$, em que $53,30 \%$ do total de mulheres com ASC-US retornaram para a citopatologia de seguimento.

O INCA/MS preconiza que, diante de resultado citopatológico de ASC-US/LSIL, deve-se repetir o exame citopatológico após seis meses, pois a maioria dessas alteraçóes regride espontaneamente; no entanto, caso se mantenha o mesmo resultado ou pior diagnóstico, a mulher deve ser encaminhada à UMC para realizar o exame colposcópico com biópsia dirigida ${ }^{15}$. Entretanto, 
Tabela 2. Resultado do seguimento de mulheres com exames citopatológicos classificados como ASC-US/LSIL encaminhadas ao serviço de atenção secundária, no município de Goiânia, no período de março de 2006 a maio de 2008

\begin{tabular}{|c|c|c|c|c|c|c|}
\hline $\begin{array}{l}\text { Resultado da } \\
\text { citopatologia de } \\
\text { encaminhamento }\end{array}$ & $\begin{array}{l}\text { Resultado da } \\
\text { citopatologia } \\
\text { de seguimento }\end{array}$ & n (\%) & $\begin{array}{l}\text { Resultado da } \\
\text { colposcopia }\end{array}$ & n (\%) & $\begin{array}{l}\text { Resultado da } \\
\text { biópsia }\end{array}$ & n (\%) \\
\hline \multirow{8}{*}{$\begin{array}{l}\text { ASC-US/LSIL } \\
(n=579)\end{array}$} & Insatisfatório & $3(1,24)$ & Insatisfatório & $2(2,33)$ & $\begin{array}{c}\text { Cervicite crônica } \\
\text { e metaplasia } \\
\text { escamosa }\end{array}$ & $13(22,03)$ \\
\hline & Negativo & $138(57,56)$ & Alterada & $61(70,93)$ & NIC I & $27(45,76)$ \\
\hline & ASC-US/LSIL & $66(27,39)$ & Normal & $23(26,74)$ & NIC II/III & $19(32,20)$ \\
\hline & ASC-H/HSIL & $34(14,11)$ & Total & $86(14,85)$ & Adeno in situ & $0(0,00)$ \\
\hline & $\begin{array}{l}\text { AGC/Adeno in } \\
\text { situ }\end{array}$ & $0(0,00)$ & & & Ca inv & $0(0,00)$ \\
\hline & $\begin{array}{c}\text { Ca inv/ Adeno } \\
\text { inv }\end{array}$ & $0(0,00)$ & & & Adeno inv & $0(0,00)$ \\
\hline & Total & $241(41,62)$ & & & Total & $59(10,19)$ \\
\hline & $\begin{array}{c}\text { Seguimento } \\
\text { Ignorado }\end{array}$ & $338(58,38)$ & & & & \\
\hline
\end{tabular}

ASC-US: células escamosas atípicas de significado indeterminado, possivelmente não neoplásicas; LSIL: lesão intraepitelial de baixo grau; ASC-H: células escamosas atípicas de significado indeterminado, não podendo excluir lesão de alto grau; HSIL: lesão intraepitelial de alto grau; AGC: células glandulares atípicas; adeno in situ: adenocarcinoma in situ; Ca inv: carcinoma escamoso invasor; adeno inv: adenocarcinoma invasor; NIC I: neoplasia intraepitelial grau I; NIC II/III: neoplasia intraepitelial graus II e III.

Tabela 3. Resultado do seguimento de mulheres com exames citopatológicos classificados como ASC-H/HSIL e AGC/Adeno in situ encaminhadas ao serviço de atenção secundária, no município de Goiânia, no período de março de 2006 a maio de 2008

\begin{tabular}{|c|c|c|c|c|c|c|}
\hline $\begin{array}{l}\text { Resultado da } \\
\text { citopatologia de } \\
\text { encaminhamento }\end{array}$ & $\begin{array}{l}\text { Resultado da } \\
\text { citopatologia } \\
\text { de seguimento }\end{array}$ & n (\%) & $\begin{array}{l}\text { Resultado da } \\
\text { colposcopia }\end{array}$ & n (\%) & $\begin{array}{l}\text { Resultado da } \\
\text { biópsia }\end{array}$ & n (\%) \\
\hline \multirow{7}{*}{$\begin{array}{l}\text { ASC-H/HSIL } \\
(n=297)\end{array}$} & Insatisfatório & $0(0,00)$ & Insatisfatório & $5(4,81)$ & $\begin{array}{c}\text { Cervicite crônica } \\
\text { e metaplasia } \\
\text { escamosa }\end{array}$ & $15(17,44)$ \\
\hline & Negativo & $58(45,67)$ & Alterada & $79(75,96)$ & NIC I & $30(34,88)$ \\
\hline & ASC-US/LSIL & $24(18,90)$ & Normal & $20(19,23)$ & NIC II/III & $39(45,35)$ \\
\hline & ASC-H/HSIL & $43(33,86)$ & Total & $\begin{array}{c}104 \\
(35,02)\end{array}$ & Adeno in situ & $1(1,16)$ \\
\hline & $\begin{array}{l}\text { AGC/Adeno in } \\
\text { situ }\end{array}$ & $0(0,00)$ & $\begin{array}{l}\text { Seguimento } \\
\text { ignorado }\end{array}$ & $\begin{array}{c}193 \\
(64,98)\end{array}$ & Ca inv & $1(1,16)$ \\
\hline & $\begin{array}{c}\text { Ca inv/ Adeno } \\
\text { inv }\end{array}$ & $2(1,57)$ & & & Adeno inv & $0(0,00)$ \\
\hline & Total & $127(42,76)$ & & & Total & $86(28,96)$ \\
\hline \multirow{7}{*}{$\begin{array}{l}\text { AGC/Adeno in situ } \\
(n=24)\end{array}$} & Insatisfatório & $0(0,00)$ & Insatisfatório & $1(25,00)$ & $\begin{array}{c}\text { Cervicite crônica } \\
\text { e metaplasia } \\
\text { escamosa }\end{array}$ & $1(33,33)$ \\
\hline & Negativo & $2(40,00)$ & Alterada & $3(75,00)$ & NIC I & $1(33,33)$ \\
\hline & ASC-US/LSIL & $0(0,00)$ & Normal & $0(0,00)$ & NIC II/III & $1(33,33)$ \\
\hline & ASC-H/HSIL & $2(40,00)$ & Total & $4(16,67)$ & Adeno in situ & $0(0,00)$ \\
\hline & $\begin{array}{l}\text { AGC/Adeno in } \\
\text { situ }\end{array}$ & $1(20,00)$ & $\begin{array}{l}\text { Seguimento } \\
\text { ignorado }\end{array}$ & $20(83,33)$ & Ca inv & $0(0,00)$ \\
\hline & $\begin{array}{c}\text { Ca inv/ Adeno } \\
\text { inv }\end{array}$ & $0(0,00)$ & & & Adeno inv & $0(0,00)$ \\
\hline & Total & $5(20,83)$ & & & Total & $3(12,50)$ \\
\hline
\end{tabular}

ASC-US: células escamosas atípicas de significado indeterminado, possivelmente nẫo neoplásicas; LSIL: lesão intraepitelial de baixo grau; ASC-H: células escamosas atípicas de significado indeterminado, não podendo excluir lesão de alto grau; HSIL: lesão intraepitelial de alto grau; AGC: células glandulares atípicas; adeno in situ: adenocarcinoma in situ; Ca inv: carcinoma escamoso invasor; adeno inv: adenocarcinoma invasor; NIC I: neoplasia intraepitelial grau I; NIC II/III: neoplasia intraepitelial graus II e III. 
há controvérsia quanto a essa conduta, Veiga et al. ${ }^{20}$ mostraram que a prevalência de lesóes precursoras e câncer cervical encontrada em mulheres com resultados citopatológicos classificados como ASC-US com intervalo de seis meses é de cerca de $12,00 \%$, e que o encaminhamento para colposcopia somente depois dos dois resultados poderia retardar o diagnóstico. Neste estudo, observou-se que aproximadamente um terço das mulheres com ASC-US/LSIL que foram submetidas à biópsia apresentou resultado de NIC II/III.

Em relação às mulheres com resultados citopatológicos classificados como ASC-H/HSIL, 127 (42,76\%) repetiram o exame citopatológico desnecessariamente, uma vez que todas deveriam ter sido encaminhadas imediatamente para o exame colposcópico. Essa conduta acarretou atraso na confirmação do diagnóstico e, consequentemente, do tratamento, de acordo com o preconizado pelo MS, além de gastos desnecessários com a realização da citopatologia de seguimento. Essa conduta equivocada também foi detectada por Albuquerque et al. ${ }^{13}$ que constataram que $70,00 \%$ das mulheres com resultados citopatológicos alterados classificados como ASC-H/HSIL foram submetidas ao exame colposcópico.

A colposcopia subsequente ao resultado citopatológico é a melhor forma de identificar as atipias que correspondem à NIC de alto grau e as separar daquelas que não apresentam alteraçóes intraepiteliais ${ }^{21}$. Por esse motivo, a observaçáo das normas e os encaminhamentos corretos são parâmetros importantes para o melhor desempenho do programa de rastreamento e controle do câncer do colo do útero, excluindo assim procedimentos e condutas inadequadas, que podem gerar custos adicionais ${ }^{6}$. Além de ser uma alternativa econômica, a conduta preconizada é viável e simples, podendo ser realizada em consultório ginecológico e $\mathrm{UABS}^{22}$.

Ainda neste estudo, observou-se que, das mulheres com resultados citopatólogicos classificados como ASC-H/ HSIL que foram submetidas à biópsia, 39 (45,35\%) tiveram o diagnóstico de NIC II/III. Isso sugere que, entre as mulheres que náo realizaram a colposcopia, grande parte poderia apresentar diagnóstico alterado no exame histopatológico, destacando um possível atraso no diagnóstico.

Em relaçáo às mulheres com resultados citopatológicos classificados como AGC/adeno in situ, observou-se também a realização desnecessária do exame citopatológico de seguimento. A recomendação é de que todas as mulheres com esse resultado devem ser encaminhadas para exame colposcópico e biópsia. No entanto, neste estudo, observou-se que apenas $4(16,67 \%)$ dessas mulheres foram encaminhadas corretamente. Esse resultado foi inferior ao apresentado na literatura, no qual $75,00 \%$ das mulheres com resultados citopatológicos classificados como AGC/ adeno in situ foram submetidas à colposcopia ${ }^{13}$.
O presente estudo mostrou uma grande limitação devido à falta de preenchimento correto dos prontuários dificultando a identificaçáo das condutas preconizadas em grande parte dos exames realizados ${ }^{23}$. Observou-se que, do total de 900 mulheres com exames citopatológicos alterados, $551(61,22 \%)$ tiveram seguimento ignorado, possivelmente não foram orientadas e encaminhadas conforme as normas preconizadas pelo MS; por outro lado, outros motivos podem estar relacionados à própria mulher, como a situação de trabalho ou dificuldades financeiras e de locomoção, ocasionando gastos desnecessários ao SUS e também retardo no tratamento de lesões mais graves ${ }^{24,25}$.

Vale et al. ${ }^{10}$ observaram que, ao longo de sete anos, cerca de $64 \%$ dos exames foram considerados em excesso, por não seguirem as normas recomendadas. Portanto, é preciso diminuir o número de procedimentos em excesso e otimizar os recursos disponíveis, porém isso não significa redução do número de coletas do exame citopatológico, mas sim o desenvolvimento de estratégias para que as mulheres com maior risco de desenvolverem câncer e com pouca frequência de realização de exames de prevenção tenham acesso ao sistema.

Para superar esse problema, é necessário orientar as mulheres, fazendo com que elas possam compreender a importância da prevenção do câncer do colo do útero e o seguimento conforme recomendaçóes preconizadas, bem como melhorar a interação profissional-paciente e aperfeiçoar a operacionalização do serviço de acordo com as recomendaçóes do MS.

\section{CONCLUSÃO}

Conclui-se que $551(61,22 \%)$ mulheres com exames citopatológicos alterados tiveram seguimento ignorado, que possivelmente não foram submetidas às condutas preconizadas pelo MS para rastreamento do câncer do colo do útero, caracterizando assim uma grande preocupação pelo aumento de custos com a realização de exames desnecessários e o retardo do diagnóstico e tratamento de lesôes precursoras.

\section{AGRADECIMENTOS}

Agradecemos a colaboração da equipe de profissionais do Laboratório de Análises Clínicas Rômulo Rocha, da Faculdade de Farmácia, da Universidade Federal de Goiás.

\section{CONTRIBUIÇÕES}

Eduardo Silva Araújo e Flávia Moreira Barbosa contribuíram na concepçáo, planejamento da pesquisa, na redação, análise e interpretação dos dados. Cinara Zago Silveira Ázara e Tatyana Xavier Almeida Matteucci Ferreira contribuíram na redação e revisão crítica do artigo. 
Suelene Brito do Nascimento Tavares e Rita Goreti Amaral contribuíram na concepção e planejamento da pesquisa, na redação e revisão crítica do artigo.

\section{Declaraçáo de Conflito de Interesses: Nada a Declarar.}

\section{REFERÊNCIAS}

1. Uchimura NS, Nakano K, Nakano LCG, Uchimura TT. Qualidade e desempenho das citopatologias na prevenção e câncer de colo uterino. Rev Assoc Med Bras. 2009; 55(5):569-74.

2. Instituto Nacional de Câncer José Alencar Gomes da Silva. Estimativa 2012: incidência de câncer no Brasil. Rio de Janeiro: INCA; 2013.

3. Bosch F, Schiffman M, Solomon D. Introducion: future research directions in the epidemiology of huma papilomavirus and cancer. J Natl Inst Monogr. 2003; 2003(31):1-2.

4. Hildesheim A, Wang SS. Host and viral genetics and risk of cervical cancer: a review. Virus Res. 2002; 89(2):229-40.

5. Tavares SBN, Amaral RG, Manrique EJC, Sousa NLA, Albuquerque ZBP, Zeferino LC. Controle da qualidade em citopatologia cervical: revisão de literatura. Rev Bras Cancerol. 2007; 53(3):355-64.

6. Instituto Nacional do Câncer (Brasil). Plano de ação para redução da incidência e mortalidade por câncer do colo do útero: sumário executivo. Rio de Janeiro: INCA; 2010.

7. Instituto Nacional do Câncer (Brasil). Diretrizes brasileiras para o rastreamento do câncer do cólo do útero. Rio de Janeiro: INCA; 2011.

8. Girianelli VR, Thuler LCS, Silva GA. Qualidade do sistema de informação do câncer do colo do útero no estado do Rio de Janeiro. Rev Saúde Pública. 2009; 43(4):580-8.

9. Madlensky L, Goel V, Polzer J, Ashbury FD. Assessing the evidence for organised cancer screening programmes. Eur J Cancer. 2003; 39(12):1648-53.

10. Vale DBAP, Morais SS, Pimenta AL, Zeferino LC. Avaliação do rastreamento do câncer do colo do útero na Estratégia Saúde da Família no Município de Amparo, São Paulo, Brasil. Cad Saúde Pública. 2010; 26(2):383-90.

11.Zeferino LC. The challenge of reducing mortality due to cervical cancer. Rev Bras Ginecol Obstet. 2008; 30(5):213-5.

12. Goldie SJ, Gaffikin L, Goldhaber-Fiebert JD, GordilloTobar A, Levin C, Mahé C, et al. Cost-effectiveness of cervical-cancer screening in five developing countries. $\mathrm{N}$ Engl J Med. 2005; 353(20):2158-68.

13. Albuquerque ZBP, Manrique EJC, Tavares SBN, Souza ACS, Guimarães JV, Amaral RB. Mulheres com atipias, lesōes precursoras e invasivas do colo do útero: condutas segundo as recomendações do Ministério da Saúde. Rev Bras Ginecol Obstet. 2012; 34(6):258-63.

14. Ministério da Saúde (Brasil). Prevenção do câncer do colo do útero. Manual Técnico para Laboratórios. Brasília, DF: MS; 2002.

15. Instituto Nacional do Câncer (Brasil). Nomenclatura brasileira para laudos cervicais e condutas preconizadas: recomendações para profissionais de saúde. 2. ed. Rio de Janeiro: INCA; 2006.

16. Walker P, Dexeus S, De Palo G, Barrasso R, Campion M, Girardi F, et al. International terminology of colposcopy: an updated report. from the international federation for cervical pathology and colposcopy. Obstet Gynecol. 2003; 101(1):175-7.

17. Scully RE, Bonfiglio TA, Kurman RJ, Silverberg SG, Wilkinson EJ. Histological typing of female genital tract tumors. World Health Organizations. 2nd ed. Berlin: Springer-verlag; 1994. (World Health OrganizationsInternational Histological Classification of Tumours).

18. Centers for Disease Control and Prevention. EPI INFO. Epidemiology Program Office Division of Public Health Surveillance and informatics. Version: Epi Info TM Version 3.5.1; Aug 2008 [acesso 2009 Mar. 02]. Disponível em: http://www.cdc.gov/epiinfo/.

19. Bueno KS. Atipias escamosas de significado indeterminado: novas qualificações e importância na conduta clínica. Rev Bras Anal Clin. 2008; 40(2):121-8.

20. Veiga FR, Russomano F, Camargo MJ, Monteiro ACS, Reis A, Tristão MA. Prevalência das lesōes intra-epiteliais de alto grau em pacientes com citologia com diagnóstico persistente de ASCUS. Rev Bras Ginecol Obstet. 2006; 28(2):75-80.

21. Pedrosa ML. Perfil epidemiológico de mulheres portadoras de atipias escamosas de significado indeterminado atendidas pelo programa de controle do câncer de colo uterino no município do Rio de Janeiro [dissertação]. Rio de Janeiro: Fundação Oswaldo Cruz; 2003.

22. Uchimura NS, Uchimura TT, Martins JPOB, Assakawa F, Uchimura LYT. Avaliação da conduta conservadora na lesão intraepitelial cervical de alto grau. Rev. Saúde Pública. 2012; 46(3):466-71.

23. Santos ERR, Silva KCL, Bezerra AFB. Desafios para organização do rastreamento do câncer no colo uterino em um município da região metropolitana do Recife. Rev Ciênc Méd (Campinas). 2012; 21(1-6):45-54.

24. Greenwood SA, Machado MFAS, Sampaio NMV. Motivos que levam mulheres a não retornarem para receber o resultado de exame Papanicolau. Rev Latinoam Enfermagem. 2006; 4(4):503-9.

25. Cruz LMB, Loureiro RP. A comunicação na abordagem preventiva do câncer do colo do útero: importância das influências histórico-culturais e da sexualidade feminina na adesão às campanhas. Saude Soc. 2008; 17(2):120-31 . 


\begin{abstract}
Introduction: The appropriate follow-up for women with abnormal cytopathology tests is important for the treatment of precursor lesions in the initial phase. Objective: To assess whether women saw in Basic Health Care Units with abnormal cytopathology tests were subjected to properly conduct recommended by the Ministry of Health. Method: The study analyzed 12,208 cytopathology results of women attending Units Primary Care in Goiânia, the period 20062008, through the records of Primary Care and Medium Complexity Units. The Epi-info 3.5.1 software was used for data analysis. Results: Of the 12,208 results analyzed, $90.74 \%$ were classified as negative, $1.88 \%$ as unsatisfactory, $4.74 \%$ as less serious injuries, and $2.43 \%$ as medium severity injuries. Of the total, 579 women with cytopathology tests classified as atypical squamous cells of undetermined significance, possibly non-neoplastic/low-grade squamous intraepithelial lesion, $41.62 \%$ underwent cytology follow-up, 14.85\% and 10.19\% underwent colposcopy and biopsy, respectively. Of the 297 women with cytopathological findings classified as atypical squamous cells of undetermined significance, high-grade lesion/high-grade squamous intraepithelial lesion cannot be excluded, $42.76 \%$ underwent cytopathology follow-up, and $35.02 \%$ and $28.96 \%$ underwent colposcopy and a biopsy, respectively. Of the 24 women with cytopathological results reported as atypical glandular cells/invasive adenocarcinoma, $20.83 \%$ had follow-up cytopathology, and $16.67 \%$ and $12.50 \%$ of women underwent colposcopy and biopsy, respectively. Conclusion: The majority of women with abnormal cytopathology tests possibly were not subjected to the recommended protocols as recommended by the Ministry of Health.

Key words: Female; Uterine Cervical Neoplasms-diagnosis; Uterine Cervical Neoplasms-epidemiology; Primary Health Care; Mass Screening-utilization; Brazil-epidemiology
\end{abstract}

\title{
Resumen
}

Introducción: El seguimiento adecuado a las mujeres con alteraciones citopatológicas es importante para el tratamiento de lesiones precursoras cuando se encuentran en la fase inicial. Objetivo: Evaluar si las mujeres atendidas en las Unidades de Atención Básica de Salud con alteraciones en análisis citopatológicos fueron sometidas adecuadamente a procedimientos recomendados por el Ministerio de Salud de Brasil. Método: Fueron analizados 12.208 resultados de exámenes citopatológicos de mujeres atendidas en Unidades de Atención Básica de Salud en la ciudad de Goiania, desde 2006 hasta 2008, utilizando el historial clínico de pacientes de las Unidades de Atención Básica y de Media complejidad. Fue usado el programa Epi-info 3.5.1 para análisis de datos. Resultados: De los 12.208 resultados analizados, el $90,74 \%$ fue clasificado como negativo, el 1,88\% como insatisfactorio, el 4,74\% como lesiones menos graves y el 2,43\% como lesiones más graves. Un total de 579 mujeres con resultados citopatológicos clasificados como células escamosas atípicas de significado indeterminado, posiblemente no neoplásicas/lesión intra-epitelial escamosa de menor grado, el $41,62 \%$ se sometió a citopatología de seguimiento, $14,85 \%$ y $10,19 \%$ se sometieron a colposcopia y biopsia respectivamente. De las 297 mujeres con resultados citopatológicos clasificados como células escamosas atípicas de significado indeterminado, no se puede excluir lesión de alto grado/lesión intra-epitelial escamosa de alto grado, $42,76 \%$ tenían seguimiento citopatológico, $35,02 \%$ y $28,96 \%$ se sometieron a colposcopia y biopsia respectivamente. De las 24 mujeres con resultado citopatológico clasificados como células glandulares atípicas/adenocarcinoma invasivo, $20,83 \%$ tenían seguimiento citopatológico, $16,67 \%$ y $12,50 \%$ se sometieron a colposcopia y biopsia, respectivamente. Conclusión: La mayoría de las mujeres con alteraciones en los exámenes citopatológicos probablemente no se sometieron a los procedimientos recomendados por el Ministerio de Salud de Brasil.

Palabras clave: Femenino; Neoplasias del Cuello do Uterino- diagnóstico; Neoplasias del Cuello do Uterinoepidemiología; Atención Primaria de Salud; Tamizaje Masivo-utilización; Brasil-epidemiologia 